\title{
Asymptotic behavior of a free boundary problem for the growth of multi-layer tumors in necrotic phase
}

\author{
Junde $\mathrm{Wu}$ \\ Department of Mathematics, Soochow University, Suzhou, \\ Jiangsu 215006, PR China. E-mail: wujund@suda.edu.cn
}

\begin{abstract}
In this paper we study a free boundary problem for the growth of multi-layer tumors in necrotic phase. The tumor region is strip-like and divided into necrotic region and proliferating region with two free boundaries. The upper free boundary is tumor surface and governed by a Stefan condition. The lower free boundary is the interface separating necrotic region from proliferating region, its evolution is implicit and intrinsically governed by an obstacle problem. We prove that the problem has a unique flat stationary solution, and there exists a positive constant $\gamma_{*}$, such that the flat stationary solution is asymptotically stable for cell-to-cell adhesiveness $\gamma>\gamma_{*}$, and unstable for $0<\gamma<\gamma_{*}$.
\end{abstract}

2010 Mathematics Subject Classification: 35B40; 35K55; 35Q92; 35R35

Keywords: free boundary problem; asymptotic behavior; necrotic tumor

\section{Introduction}

In this paper, we study the following free boundary problem modeling tumor growth in necrotic phase:

$$
\left\{\begin{aligned}
\Delta \sigma & =\sigma \chi_{\Omega^{+}(t)} & & \text { in } \Omega^{+}(t) \cup \Omega^{-}(t), t>0, \\
\Delta p & =-\mu(\sigma-\tilde{\sigma}) \chi_{\Omega^{+}(t)}+\nu \chi_{\Omega^{-}(t)} & & \text { in } \Omega^{+}(t) \cup \Omega^{-}(t), t>0, \\
\sigma & =\bar{\sigma}, \quad p=\gamma \kappa & & \text { on } \Gamma^{+}(t), t>0, \\
\sigma & =\hat{\sigma}, \quad \llbracket \partial_{n} \sigma \rrbracket=0 & & \text { on } \Gamma^{-}(t), t>0, \\
\llbracket p \rrbracket & =0, \quad \llbracket \partial_{n} p \rrbracket=0 & & \text { on } \Gamma^{-}(t), t>0, \\
\partial_{y} \sigma & =0, \quad \partial_{y} p=0 & & \text { on } \Gamma_{0}, t>0, \\
V & =-\partial_{n} p & & \text { on } \Gamma^{+}(t), t>0, \\
\Gamma^{+}(0) & =\Gamma_{0}^{+} & & \text {at } t=0,
\end{aligned}\right.
$$


where $\sigma=\sigma(x, y, t)$ and $p=p(x, y, t)$ are unknown functions representing concentration of nutrient and internal pressure within tumor, respectively, $\Omega^{+}(t)$ and $\Omega^{-}(t)$ are unknown domains occupied by tumor proliferating cells and necrotic cells at time $t>0$, respectively, and

$$
\begin{gathered}
\Omega^{+}(t):=\left\{(x, y) \in \mathbb{R}^{n-1} \times \mathbb{R}: \eta(x, t)<y<\rho(x, t)\right\}, \\
\Omega^{-}(t):=\left\{(x, y) \in \mathbb{R}^{n-1} \times \mathbb{R}: 0<y<\eta(x, t)\right\},
\end{gathered}
$$

where $\eta(x, t)$ and $\rho(x, t)$ are unknown functions satisfying $0<\eta(x, t)<\rho(x, t)$ for $x \in \mathbb{R}^{n-1}$ and $t>0, \Gamma^{+}(t)$ and $\Gamma^{-}(t)$ are free boundaries, and

$$
\begin{aligned}
& \Gamma^{+}(t):=\operatorname{graph}(\rho(x, t))=\left\{(x, y) \in \mathbb{R}^{n-1} \times \mathbb{R}: y=\rho(x, t)\right\}, \\
& \Gamma^{-}(t):=\operatorname{graph}(\eta(x, t))=\left\{(x, y) \in \mathbb{R}^{n-1} \times \mathbb{R}: y=\eta(x, t)\right\},
\end{aligned}
$$

$\Gamma_{0}:=\operatorname{graph}(0)$ is the fixed bottom boundary, $\kappa$ is the mean curvature and $V$ is the outward normal velocity of upper tumor surface $\Gamma^{+}(t)$, respectively, $\partial_{n}$ denotes the outward normal derivative with respect to $\Omega^{+}(t), \bar{\sigma}, \tilde{\sigma}, \hat{\sigma}, \mu, \nu$ and $\gamma$ are positive constants, where $\bar{\sigma}$ represents constant external nutrient supply, $\tilde{\sigma}$ is a critical value for the balance of cell apoptosis and mitosis, $\hat{\sigma}$ is the nutrient level for tumor cell necrosis, $\mu$ is the proliferation rate of tumor proliferating cells, $\nu$ is the dissolution rate of necrotic cells, and $\gamma$ is cell-to-cell adhesiveness. We assume that $0<\hat{\sigma}<\tilde{\sigma}<\bar{\sigma} \cdot \chi_{\Omega^{ \pm}(t)}$ is the characteristic function of $\Omega^{ \pm}(t)$, respectively. The notation $\llbracket p \rrbracket$ denotes the jump of $p$ across $\Gamma^{-}(t)$, and

$$
\llbracket p \rrbracket:=\Upsilon p^{+}-\Upsilon p^{-} \quad \text { for } \quad p^{+}=\left.p\right|_{\Omega^{+}(t)} \quad \text { and } \quad p^{-}=\left.p\right|_{\Omega^{-}(t)},
$$

where $\Upsilon$ is the trace operator on $\Gamma^{-}(t)$. Similarly, $\llbracket \partial_{n} p \rrbracket$ and $\llbracket \partial_{n} \sigma \rrbracket$ denote the jump of the normal derivatives of $p$ and $\sigma$ across $\Gamma^{-}(t)$, respectively.

Problem (1.1) is originated from mathematical model proposed by Byrne and Chaplain [3, for the growth of necrotic tumor in vitro which is cultivated on an impermeable support membrane, and tumor cells are multilayered. The first equation describes the diffusion and consumption of nutrient in tumor region; the second equation is based on Darcy's law and mass conservation law; the third equation means constant nutrient supply and pressure is continuous across the upper tumor surface, by taking cell-to-cell adhesiveness into account; the later three lines of equations mean that nutrient concentration, pressure and their normal derivatives are continuous across the lower free boundary, nutrient and tumor cells can not pass through the bottom boundary. For more details we refer to 3 .

In the non-necrotic case, i.e., $\Omega_{-}(t)=\emptyset$, the corresponding problem of (1.1) has been well studied. Cui and Escher [8] established local well-posedness and asymptotic stability of the unique flat equilibrium (independent of $x$ ). Zhou et al. 23] proved that there exist infinitely many bifurcation stationary solutions. It is worthy to mention that another extensively studied model is solid tumor spheroid model, where tumor region is sphere-shaped. For similar solid tumor spheroid models, many illuminative results such as global well-posedness, existence of bifurcation stationary solutions and Hopf bifurcations, and asymptotical stability of radially 
symmetric equilibrium have been established, we refer to [5, 6, 7, 10, 11, 12, 13, 14, 16, 20, 21, 22 and references therein.

In the necrotic case, we observe that problem (1.1) has two free boundaries. The evolution of the upper free boundary $\Gamma^{+}(t)$ is governed by the equation $V=-\partial_{n} p$, but the evolution of the lower free boundary $\Gamma^{-}(t)$ is implicit. This is a remarkable feature and make the analysis of problem (1.1) in high dimension is much more difficult than the non-necrotic case. By the maximum principle, since $0<\hat{\sigma}<\bar{\sigma}$, we have $\sigma(x, y, t) \equiv \hat{\sigma}$ in $\Omega^{-}(t)$, and $\sigma(x, y, t)>\hat{\sigma}$ in $\Omega^{+}(t)$ at each $t>0$. Let $\Omega(t):=\left\{(x, y) \in \mathbb{R}^{n-1} \times \mathbb{R}: 0<y<\rho(x, t)\right\}$ for a given function $\rho(x, t)$, we can rewrite $\Omega^{+}(t)=\{(x, y) \in \Omega(t): \sigma(x, y, t)>\hat{\sigma}\}, \Omega^{-}(t)=\operatorname{int}\{(x, y) \in \Omega(t): \sigma(x, y, t)=\hat{\sigma}\}$ and $\Gamma^{-}(t)=\partial \Omega^{+}(t) \cap \partial \Omega^{-}(t)$. We see that $\left(\sigma(x, y, t), \Gamma^{-}(t)\right)$ satisfies an obstacle problem:

$$
\left\{\begin{array}{lll}
-\Delta \sigma+\sigma \geq 0, \quad \sigma \geq \hat{\sigma}, & (-\Delta \sigma+\sigma)(\sigma-\hat{\sigma})=0 \quad \text { in } \Omega(t), \\
\sigma=\bar{\sigma} \quad \text { on } \quad \Gamma^{+}(t), & \partial_{y} \sigma=0 \quad \text { on } \Gamma_{0} .
\end{array}\right.
$$

The regularity of free boundary of obstacle problems in high dimension is very difficult to study (cf. 4, 17). Even for smooth domain $\Omega(t)$, the solution $\sigma \notin C^{2}(\Omega(t))$. It makes a main difficulty arising in necrotic tumor model from non-necrotic case. To solve this problem, we first show there is a unique flat stationary solution (see Section 2). It implies that we can consider obstacle problem (1.2) in a small neighborhood of the flat stationary solution. Motivated by Cui [5] and Hamilton [15, by using Nash-Moser implicit function theorem, for given $\Gamma^{+}(t)=\operatorname{graph}(\rho(x, t))$ closed to the flat equilibrium, we prove that the solution $\left(\sigma(x, y, t), \Gamma^{-}(t)\right)$ is smoothly depending on $\rho(x, t)$, and $\Gamma^{-}(t)$ is actually smooth in space variables (see Lemma 3.2). Then we further solve the first six lines of equations of problem (1.1) and get the solution $p(x, y, t)$ which also smoothly depends on $\rho(x, t)$, finally by the second last equation $V=-\partial_{n} p$ we reduce problem (1.1) into an abstract differential equation $\partial_{t} \rho+\Psi(\rho)=0$ only containing function $\rho(x, t)$. In suitable Banach spaces, we show this abstract differential equation is of parabolic type and the local well-posedness follows by geometric theory of parabolic differential equations. By a delicate analysis and computation, we study the spectrum of the linearized operator at the flat stationary solution, and by linearized stability principle we can get asymptotic stability of the flat stationary solution.

To give a precise statement of our main results, we introduce some notations.

In this paper, we only consider the case $n=2$, and the higher-dimensional case can be treated similarly. We denote the solution of problem (1.1) by $(\sigma, p, \eta, \rho)$, with $\Gamma^{-}(t)=$ $\operatorname{graph}(\eta(x, t))$ and $\Gamma^{+}(t)=\operatorname{graph}(\rho(x, t))$. For the sake of simplicity, we impose that

$$
\sigma(x, y, t), p(x, y, t), \eta(x, t), \rho(x, t) \text { are } 2 \pi \text {-periodic in } x \in \mathbb{R} \text {. }
$$

We identify $\mathbb{S}=\mathbb{R} / 2 \pi \mathbb{Z}$, and identify continuous $2 \pi$-periodic function space $C_{\text {per }}(\mathbb{R})=C(\mathbb{S})$. Given $s>0$, we denote by $B U C^{s}(\mathbb{S})$ the space of all bounded and uniformly Hölder continuous functions on $\mathbb{S}$ of order $s>0$. Let $h^{s}(\mathbb{S})$ denote the little Hölder space, a closure of $B U C^{\infty}(\mathbb{S})$ in $B U C^{s}(\mathbb{S})$. Similarly, we denote by $h^{s}(\Omega)$ the closure of $B U C^{\infty}(\Omega)$ in $B U C^{s}(\Omega)$ for bounded open domain $\Omega$ in $\mathbb{R}^{2}$. 
Our first main result is stated as follows:

Theorem 1.1 Let $\tilde{\sigma}>\hat{\sigma}>0$ be given. There exists a positive constant $\sigma_{*}>\tilde{\sigma}$ depending only on $\hat{\sigma}$ and $\tilde{\sigma}$, such that free boundary problem (1.1) has a unique flat stationary solution $\left(\sigma_{s}, p_{s}, \eta_{s}, \rho_{s}\right)$ if and only if $\bar{\sigma}>\sigma_{*}$.

We shall prove this result in Section 2. Recall that in non-necrotic case (see Theorem 2 of [8]), there exists a unique non-necrotic flat stationary solution for all $\bar{\sigma}>\tilde{\sigma}$. It is an interesting difference that necrotic flat stationary solution does not exist for $\tilde{\sigma}<\bar{\sigma} \leq \sigma_{*}$.

Our second main result is about asymptotic stability of the flat stationary solution.

Theorem 1.2 (i) There exists a positive threshold value $\gamma_{*}$ of cell-to-cell adhesiveness such that for any $\gamma>\gamma_{*}$, the flat stationary solution $\left(\sigma_{s}, p_{s}, \eta_{s}, \rho_{s}\right)$ is asymptotically stable in the following sense: There exists a constant $\epsilon>0$ such that if $\rho_{0} \in h^{4+\alpha}(\mathbb{S}),\left\|\rho_{0}\right\|_{h^{4+\alpha}(\mathbb{S})}<\epsilon$ and $\Gamma_{0}^{+}=\operatorname{graph}\left(\rho_{s}+\rho_{0}\right)$, then the solution $(\sigma, p, \eta, \rho)$ of problem (1.1) exists for all $t>0$ and converges to $\left(\sigma_{s}, p_{s}, \eta_{s}, \rho_{s}\right)$ exponentially fast as $t \rightarrow+\infty$.

(ii) If $0<\gamma<\gamma_{*}$, then $\left(\sigma_{s}, p_{s}, \eta_{s}, \rho_{s}\right)$ is unstable.

The above result implies that cell-to-cell adhesiveness $\gamma$ plays an important role on tumor's stability. A smaller value of $\gamma$ may make tumor more aggressive. The threshold value $\gamma_{*}$ of cell-to-cell adhesiveness is given by (4.19) and (4.21), and $\gamma_{*}$ can be regarded as a function of the dissolution rate $\nu$. By $d \gamma_{*} / d \nu \leq 0$, we see that a smaller value of $\nu$ may make tumor more unaggressive. While in the limiting case $\nu=0$, the flat stationary solution is not asymptotically stable for all $\gamma>0$. (see Remark 5.2).

The structure of the rest of this paper is arranged as follows. In the next section, we study the existence and uniqueness of flat stationary solution. In Section 3, by using implicit function theorem and classical theory of elliptic equations we reduce free boundary problem (1.1) into a Cauchy problem in little Hölder spaces, and establish the local well-posedness. Section 4 is devoted to study the linearized problem at flat stationary solution and compute eigenvalues. In the last section we make stability analysis and give a proof of Theorem 1.2.

\section{Flat stationary solution}

In this section, we study the existence and uniqueness of flat stationary solution of free boundary problem (1.1).

We denote flat stationary solution by $\left(\sigma_{s}(y), p_{s}(y), \eta_{s}, \rho_{s}\right)$ with $0<\eta_{s}<\rho_{s}$. It satisfies the following problem 


$$
\left\{\begin{array}{rlrl}
\sigma_{s}^{\prime \prime}(y)=\sigma_{s}(y), & p_{s}^{\prime \prime}(y)=-\mu\left(\sigma_{s}(y)-\tilde{\sigma}\right) & & \text { for } \eta_{s}<y<\rho_{s} \\
\sigma_{s}^{\prime \prime}(y)=0, & & \text { for } 0<y<\eta_{s} \\
\sigma_{s}\left(\rho_{s}\right)=\bar{\sigma}, & & p_{s}\left(\rho_{s}\right)=0, & \\
\sigma_{s}\left(\eta_{s}\right)=\hat{\sigma}, & \sigma_{s}^{\prime}\left(\eta_{s}\right)=0 \\
p_{s}\left(\eta_{s}^{+}\right)=p_{s}\left(\eta_{s}^{-}\right), & p_{s}^{\prime}\left(\eta_{s}^{+}\right)=p_{s}^{\prime}\left(\eta_{s}^{-}\right), & \\
\sigma_{s}^{\prime}(0)=0, & p_{s}^{\prime}(0)=0, \quad p_{s}^{\prime}\left(\rho_{s}\right)=0 .
\end{array}\right.
$$

We easily get that

$$
\begin{aligned}
& \sigma_{s}(y)= \begin{cases}\frac{\bar{\sigma} \sinh \left(y-\eta_{s}\right)+\hat{\sigma} \sinh \left(\rho_{s}-y\right)}{\sinh \left(\rho_{s}-\eta_{s}\right)} & \text { for } \eta_{s} \leq y \leq \rho_{s}, \\
\hat{\sigma} & \text { for } 0<y<\eta_{s},\end{cases} \\
& p_{s}(y)= \begin{cases}\frac{\mu}{2} \tilde{\sigma}\left(y^{2}-\rho_{s}^{2}\right)+(\nu-\mu \tilde{\sigma})\left(y-\rho_{s}\right) \eta_{s}+\mu\left(\bar{\sigma}-\sigma_{s}(y)\right) & \text { for } \eta_{s} \leq y \leq \rho_{s}, \\
\frac{\nu}{2}\left(y^{2}-\eta_{s}^{2}\right)+p_{0} & \text { for } 0<y<\eta_{s},\end{cases}
\end{aligned}
$$

where $p_{0}=\frac{\mu}{2} \tilde{\sigma}\left(\eta_{s}^{2}-\rho_{s}^{2}\right)+(\nu-\mu \tilde{\sigma})\left(\eta_{s}-\rho_{s}\right) \eta_{s}+\mu(\bar{\sigma}-\hat{\sigma})$.

By $\sigma_{s}^{\prime}\left(\eta_{s}\right)=0$, there holds

$$
\cosh \left(\rho_{s}-\eta_{s}\right)=\frac{\bar{\sigma}}{\hat{\sigma}}
$$

Using this formula,

$$
\sigma_{s}^{\prime}\left(\rho_{s}\right)=\frac{\bar{\sigma} \cosh \left(\rho_{s}-\eta_{s}\right)-\hat{\sigma}}{\sinh \left(\rho_{s}-\eta_{s}\right)}=\sqrt{\bar{\sigma}^{2}-\hat{\sigma}^{2}}
$$

By $p_{s}^{\prime}\left(\rho_{s}\right)=0$ we have $\mu \tilde{\sigma} \rho_{s}+(\nu-\mu \tilde{\sigma}) \eta_{s}-\mu \sigma_{s}^{\prime}\left(\rho_{s}\right)=0$. It implies that

$$
(\nu-\mu \tilde{\sigma}) \eta_{s}+\mu \tilde{\sigma} \rho_{s}=\mu \sqrt{\bar{\sigma}^{2}-\hat{\sigma}^{2}} .
$$

Then from (2.4) and (2.6), we obtain

$$
\begin{gathered}
\eta_{s}=\frac{\mu}{\nu}\left(\sqrt{\bar{\sigma}^{2}-\hat{\sigma}^{2}}-\tilde{\sigma} \ln \left(\bar{\sigma}+\sqrt{\bar{\sigma}^{2}-\hat{\sigma}^{2}}\right)+\tilde{\sigma} \ln \hat{\sigma}\right), \\
\rho_{s}=\eta_{s}+\ln \left(\bar{\sigma}+\sqrt{\bar{\sigma}^{2}-\hat{\sigma}^{2}}\right)-\ln \hat{\sigma} .
\end{gathered}
$$

Clearly, $\rho_{s}>\eta_{s}$ if and only if $0<\hat{\sigma}<\bar{\sigma}$.

Next, we only need to make sure $\eta_{s}>0$ for $0<\hat{\sigma}<\min \{\tilde{\sigma}, \bar{\sigma}\}$. Define a function

$$
f(a, r):=\sqrt{r^{2}-1}-a \ln \left(r+\sqrt{r^{2}-1}\right) \quad \text { for } r>1, a>1 .
$$

Note that

$$
\partial_{r} f(a, r)=\frac{r-a}{\sqrt{r^{2}-1}}, \quad f(a, a)<0 \quad \text { for } r>1, a>1
$$


It follows that for any $a>1$, there exists a positive constant $a_{*}>a$ such that

$$
f(a, r) \begin{cases}<0, & 1<r<a_{*}, \\ =0, & r=a_{*}, \\ >0, & r>a_{*} .\end{cases}
$$

By (2.7) we see that $\eta_{s}=\frac{\mu \hat{\sigma}}{\nu} f\left(\frac{\tilde{\sigma}}{\hat{\sigma}}, \frac{\bar{\sigma}}{\hat{\sigma}}\right)$. Recall that $0<\hat{\sigma}<\min \{\tilde{\sigma}, \bar{\sigma}\}$. We immediately obtain that there exists a positive constant $\sigma_{*}>\tilde{\sigma}$ depending only on $\tilde{\sigma}$ and $\hat{\sigma}$, such that $\eta_{s}>0$ for $\bar{\sigma}>\sigma_{*}$ and $\eta \leq 0$ for $\bar{\sigma} \leq \sigma_{*}$.

In conclusion, we have

Theorem 2.1 Assume $0<\hat{\sigma}<\tilde{\sigma}$. There exists a positive constant $\sigma_{*}>\tilde{\sigma}$ depending only on $\tilde{\sigma}$ and $\hat{\sigma}$, such that for $\bar{\sigma}>\sigma_{*}$, problem (1.1) has a unique flat stationary solution $\left(\sigma_{s}, p_{s}, \eta_{s}, \rho_{s}\right)$ given by (2.2), (2.3), (2.7) and (2.8). If $\bar{\sigma} \leq \sigma_{*}$, problem (1.1) has no flat stationary solution.

It is interesting to compare this result with non-necrotic case. From Theorem 2 of 8 , we see that there exists a unique non-necrotic flat stationary solution for $0<\tilde{\sigma}<\bar{\sigma}$. But in necrotic case, we see for $\tilde{\sigma}<\bar{\sigma} \leq \sigma_{*}$, necrotic flat stationary solution does not exist.

\section{$3 \quad$ Reduction and Well-posedness}

In this section, we reduce free boundary problem (1.1) into a Cauchy problem in little Hölder spaces, and study the local well-posedness.

First, we transform free boundary problem (1.1) into an equivalent problem on a fixed domain. Later on, we always assume $0<\hat{\sigma}<\tilde{\sigma}<\sigma_{*}<\bar{\sigma}$. By Theorem 2.1, problem (1.1) has a unique flat stationary solution $\left(\sigma_{s}, p_{s}, \eta_{s}, \rho_{s}\right)$. Denote

$$
\begin{gathered}
\Omega_{s}=\left\{(x, y) \in \mathbb{S} \times \mathbb{R}: 0<y<\rho_{s}\right\}, \quad \mathbb{D}_{s}=\left\{(x, y) \in \mathbb{S} \times \mathbb{R}: 0<y<\eta_{s}\right\}, \\
\Gamma_{s}=\mathbb{S} \times\left\{\rho_{s}\right\}, \quad J_{s}=\mathbb{S} \times\left\{\eta_{s}\right\}, \quad \Gamma_{0}=\mathbb{S} \times\{0\}, \quad \mathbb{E}_{s}=\Omega_{s} \backslash \overline{\mathbb{D}}_{s} .
\end{gathered}
$$

Let $r_{0}:=\left(\rho_{s}-\eta_{s}\right) / 8, \delta \in\left(0, r_{0}\right)$ and $\alpha \in(0,1)$, set

$$
\mathcal{O}_{\delta}:=\left\{\rho \in h^{4+\alpha}(\mathbb{S}):\|\rho\|_{h^{4+\alpha}(\mathbb{S})}<\delta\right\} .
$$

For $\rho, \eta \in \mathcal{O}_{\delta}$, we denote

$$
\begin{array}{cc}
\Omega_{\rho}=\left\{(x, y) \in \mathbb{S} \times \mathbb{R}: 0<y<\rho_{s}+\rho(x)\right\}, & \mathbb{D}_{\eta}=\left\{(x, y) \in \mathbb{S} \times \mathbb{R}: 0<y<\eta_{s}+\eta(x)\right\}, \\
\Gamma_{\rho}=\left\{(x, y) \in \mathbb{S} \times \mathbb{R}: y=\rho_{s}+\rho(x)\right\}, & J_{\eta}=\left\{(x, y) \in \mathbb{S} \times \mathbb{R}: y=\eta_{s}+\eta(x)\right\},
\end{array}
$$




$$
\Omega_{\rho, \eta}=\Omega_{\rho} \backslash \overline{\mathbb{D}}_{\eta} \quad \text { and } \quad \mathbb{E}_{\eta}=\Omega_{s} \backslash \overline{\mathbb{D}}_{\eta} .
$$

Choose a function $\varphi \in C^{\infty}(\mathbb{R})$ such that

$$
0 \leq \varphi(y) \leq 1, \quad \varphi(y)=\left\{\begin{array}{ll}
1, & \text { for }|y| \leq \delta, \\
0, & \text { for }|y| \geq 3 \delta,
\end{array} \quad \sup \left|\varphi^{\prime}(y)\right|<1 / \delta .\right.
$$

Given $\rho \in \mathcal{O}_{\delta}$, we introduce a mapping

$$
\Phi_{\rho}: \Omega_{s} \rightarrow \Omega_{\rho}, \quad(x, y) \rightarrow\left(x, y+\varphi\left(y-\rho_{s}\right) \rho(x)\right) .
$$

Clearly, $\Phi_{\rho}\left(\Omega_{s}\right)=\Omega_{\rho}, \Phi_{\rho}\left(\Gamma_{s}\right)=\Gamma_{\rho}$ and $\Phi_{\rho}$ is a $h^{4+\alpha}$ diffeomorphism from $\Omega_{s}$ onto $\Omega_{\rho}$. Moreover, for any $\eta \in \mathcal{O}_{\delta}, \Phi_{\rho}$ is the identity mapping on $\mathbb{D}_{\eta}$. Define the induced push-forward operator $\Phi_{*}^{\rho}$, and pull-back operator $\Phi_{\rho}^{*}$ by

$$
\Phi_{*}^{\rho} u=u \circ \Phi_{\rho}^{-1} \quad \text { for } u \in C\left(\Omega_{s}\right), \quad \Phi_{\rho}^{*} v=v \circ \Phi_{\rho} \quad \text { for } v \in C\left(\Omega_{\rho}\right) .
$$

Next, we introduce the following transformed operators:

$$
\mathcal{A}(\rho) u:=\Phi_{\rho}^{*} \Delta\left(\Phi_{*}^{\rho} u\right), \quad \mathcal{B}(\rho) u:=\left\langle\left.\nabla\left(\Phi_{*}^{\rho} u\right)\right|_{\Gamma_{\rho}}, \mathbf{n}_{\rho}\right\rangle \quad \text { for } u \in H^{2}\left(\Omega_{s}\right)
$$

where $\mathbf{n}_{\rho}=\left(-\rho_{x}, 1\right)$ is the outward normal on $\Gamma_{\rho},\langle\cdot, \cdot\rangle$ denotes the Euclidean inner product, and $H^{2}\left(\Omega_{s}\right)$ stands for Sobolev space. By Lemma 2.2 of [9], we have

$$
\begin{cases}\mathcal{A} \in C^{\infty}\left(\mathcal{O}_{\delta}, L\left(h^{k+2+\alpha}\left(\Omega_{s}\right), h^{k+\alpha}\left(\Omega_{s}\right)\right)\right), & 0 \leq k \leq 2, \\ \mathcal{B} \in C^{\infty}\left(\mathcal{O}_{\delta}, L\left(h^{k+1+\alpha}\left(\Omega_{s}\right), h^{k+\alpha}(\mathbb{S})\right)\right), & 0 \leq k \leq 3 .\end{cases}
$$

Denote by $\mathcal{K}(\rho)$ the transformed mean curvature on $\Gamma_{\rho}$ and

$$
\mathcal{K}(\rho)=-\left(1+\rho_{x}^{2}\right)^{-\frac{3}{2}} \rho_{x x}
$$

For some $T>0$, and a function $\rho \in C\left([0, T), \mathcal{O}_{\delta}\right) \cap C^{1}\left([0, T), h^{1+\alpha}(\mathbb{S})\right)$, we identify $\rho(x, t)=$ $\rho(t)(x)$ for $t \in[0, T)$ and $x \in \mathbb{S}$. By an elementary analysis, the outward normal velocity $V$ of tumor surface $\Gamma_{\rho(t)}$ can be given by

$$
V=\rho_{t} / \sqrt{1+\rho_{x}^{2}}
$$

Let $\chi_{\mathbb{D}_{\eta}}$ and $\chi_{\mathbb{E}_{\eta}}$ be the characteristic functions of $\mathbb{D}_{\eta}$ and $\mathbb{E}_{\eta}$, respectively. Rewrite

$$
\Gamma_{0}^{+}=\operatorname{graph}\left(\rho_{s}+\rho_{0}\right) \quad \text { for some } \rho_{0} \in \mathcal{O}_{\delta},
$$

and

$$
u(x, y, t)=\Phi_{\rho}^{*} \sigma(x, y, t), \quad v(x, y, t)=\Phi_{\rho}^{*} p(x, y, t) .
$$


One can easily check that free boundary problem (1.1) is transformed into the following problem:

$$
\left\{\begin{aligned}
\mathcal{A}(\rho) u & =u \chi_{\mathbb{E}_{\eta}} & & \text { in } \Omega_{s}, t>0, \\
\mathcal{A}(\rho) v & =-\mu(u-\tilde{\sigma}) \chi_{\mathbb{E}_{\eta}}+\nu \chi_{\mathbb{D}_{\eta}} & & \text { in } \Omega_{s}, t>0, \\
u & =\bar{\sigma}, \quad v=\gamma \mathcal{K}(\rho) & & \text { on } \Gamma_{s}, t>0, \\
u & =\hat{\sigma}, \quad \llbracket \partial_{n} u \rrbracket=0 & & \text { on } J_{\eta}, t>0, \\
\llbracket v \rrbracket & =0, \quad \llbracket \partial_{n} v \rrbracket=0 & & \text { on } J_{\eta}, t>0, \\
\partial_{y} u & =0, \quad \partial_{y} v=0 & & \text { on } \Gamma_{0}, t>0, \\
\partial_{t} \rho & =-\mathcal{B}(\rho) v & & \text { on } \mathbb{S}, t>0, \\
\rho(0) & =\rho_{0} & & \text { on } \mathbb{S}, t=0 .
\end{aligned}\right.
$$

By above transformation, we have

Lemma 3.1 A quadruple $(u, v, \eta, \rho)$ is a solution of problem (3.7) if and only if the quadruple $\left(\sigma, p, \eta_{s}+\eta, \rho_{s}+\rho\right)$ is a solution of problem (1.1) in the neighborhood of $\left(\sigma_{s}, p_{s}, \eta_{s}, \rho_{s}\right)$, with $\sigma=\Phi_{*}^{\rho} u$ and $p=\Phi_{*}^{\rho} v$.

Next we further reduce problem (3.7) into a Cauchy problem in little Hölder space for $\rho$ only. Given $\rho \in \mathcal{O}_{\delta}$, we consider the following problem:

$$
\left\{\begin{array}{l}
\mathcal{A}(\rho) u=u \chi_{\mathbb{E}_{\eta}} \quad \text { in } \Omega_{s}, \\
\left.u\right|_{\Gamma_{s}}=\bar{\sigma},\left.\quad \partial_{y} u\right|_{\Gamma_{0}}=0, \\
u=\hat{\sigma}, \quad \llbracket \partial_{n} u \rrbracket=0 \quad \text { on } J_{\eta} .
\end{array}\right.
$$

For any $\eta \in \mathcal{O}_{\delta}$, by the maximum principle, $u \equiv \hat{\sigma}$ in $\mathbb{D}_{\eta}$, and $u>\hat{\sigma}$ in $\mathbb{E}_{\eta}$. On the other hand, since $u=\hat{\sigma}$ on $J_{\eta}$, we have

$$
\partial_{n} u=\frac{u_{x} \eta_{x}-u_{y}}{\sqrt{1+\eta_{x}^{2}}}=-u_{y} \sqrt{1+\eta_{x}^{2}} \quad \text { on } J_{\eta}
$$

It implies that $\llbracket \partial_{n} u \rrbracket=0$ is equivalent to $\partial_{y} u=0$ on $J_{\eta}$. Hence for problem (3.8), we only need to solve

$$
\left\{\begin{aligned}
\mathcal{A}(\rho) u=u & \text { in } \mathbb{E}_{\eta}, \\
u=\bar{\sigma} & \text { on } \Gamma_{s}, \\
\partial_{y} u=0 & \text { on } J_{\eta}, \\
u=\hat{\sigma} & \text { on } J_{\eta} .
\end{aligned}\right.
$$

Recently, Cui [5] studied a similar obstacle problem based on Nash-Moser implicit function theorem. Motivated by this method and with some modifications to the proof of Theorem 5.2 of [5], we have the following result: 
Lemma 3.2 There exists a constant $\delta_{1} \in\left(0, r_{0}\right)$, such that for any $\rho \in \mathcal{O}_{\delta_{1}}$, problem (3.9) has a unique solution $(u, \eta)$ satisfying $u \in h^{4+\alpha}\left(\mathbb{E}_{\eta}\right)$ and $\eta \in C^{\infty}(\mathbb{S})$. Moreover, the mapping $\rho \mapsto(u, \eta)$ from $\mathcal{O}_{\delta_{1}}$ to $h^{4+\alpha}\left(\mathbb{E}_{\eta}\right) \times C^{\infty}(\mathbb{S})$ is smooth.

Proof. Denote

$$
\mathbb{H}:=\left\{(x, y) \in \mathbb{S} \times \mathbb{R}: \frac{\rho_{s}+\eta_{s}}{2}<y<\rho_{s}\right\}, \quad \mathbb{K}:=\left\{(x, y) \in \mathbb{S} \times \mathbb{R}: \eta_{s}<y<\frac{\rho_{s}+\eta_{s}}{2}\right\} .
$$

Let $r_{1}:=\min \left\{\left(\rho_{s}-\eta_{s}\right) / 8, \eta_{s} / 8\right\} \leq r_{0}$ and $\delta \in\left(0, r_{1}\right)$. For any $m \in \mathbb{N}, m \geq 4$ and $\alpha \in(0,1)$, denote

$$
\widetilde{\mathcal{O}}_{\delta}^{m+\alpha}:=\left\{\eta \in h^{m+\alpha}(\mathbb{S}):\|\eta\|_{h^{4+\alpha}(\mathbb{S})}<\delta\right\} .
$$

For any $\eta \in \widetilde{\mathcal{O}}_{\delta}^{m+\alpha}$, we introduce a mapping

$$
\widetilde{\Phi}_{\eta}: \mathbb{R}^{2} \rightarrow \mathbb{R}^{2}, \quad(x, y) \rightarrow\left(x, y+\varphi\left(y-\eta_{s}\right) \eta(x)\right),
$$

where $\varphi$ is a smooth function given by (3.2). We have $\widetilde{\Phi}_{\eta}$ is a $h^{m+\alpha}$ diffeomorphism from $\mathbb{E}_{s}$ onto $\mathbb{E}_{\eta}$, and $\widetilde{\Phi}_{\eta}$ is the identity mapping on $\mathbb{H}$. Similarly as (3.3), we can define the push-forward operator $\widetilde{\Phi}_{*}^{\eta}$, and pull-back operator $\widetilde{\Phi}_{\eta}^{*}$ induced by $\widetilde{\Phi}_{\eta}$, and for any $\rho \in \mathcal{O}_{\delta}$ and $\eta \in \widetilde{\mathcal{O}}_{\delta}^{m+\alpha}$, we define an operator

$$
\mathscr{A}(\rho, \eta) v:=\widetilde{\Phi}_{\eta}^{*} \mathcal{A}(\rho)\left(\widetilde{\Phi}_{*}^{\eta} v\right) \quad \text { for } v \in B U C^{2}\left(\mathbb{E}_{s}\right) .
$$

Notice that for any $\rho \in \mathcal{O}_{\delta}$ and $\eta \in \widetilde{\mathcal{O}}_{\delta}^{m+\alpha}, \mathcal{A}(\rho) \equiv \Delta$ in $\mathbb{K}$, we see that $\mathscr{A}(\rho, \eta)$ is independent of $\eta$ on $\mathbb{H}$, and independent of $\rho$ on $\mathbb{K}$. Moreover, $\mathscr{A}(\rho, \eta)$ is uniformly elliptic and by Lemma 2.2 in 9 , we have

$$
\mathscr{A} \in C^{\infty}\left(\mathcal{O}_{\delta} \times \widetilde{\mathcal{O}}_{\delta}^{m+\alpha}, L\left(h^{4+\alpha}\left(\mathbb{E}_{s}\right) \cap h^{m+\alpha}(\mathbb{K}), h^{2+\alpha}\left(\mathbb{E}_{s}\right) \cap h^{m-2+\alpha}(\mathbb{K})\right)\right) .
$$

Set $\tilde{u}=u \circ \widetilde{\Phi}_{\eta}$. The first three equations of (3.9) is equivalent to

$$
\mathscr{A}(\rho, \eta) \tilde{u}=\tilde{u} \quad \text { in } \mathbb{E}_{s}, \quad \tilde{u}=\bar{\sigma} \quad \text { on } \Gamma_{s}, \quad \partial_{y} \tilde{u}=0 \quad \text { on } J_{s} .
$$

By well-known regularity theory of second-order elliptic differential equations, problem (3.10) has a unique solution $\tilde{u}:=\widetilde{\mathcal{U}}(\rho, \eta) \in h^{4+\alpha}\left(\mathbb{E}_{s}\right)$, and by Lemma 2.3 in 9], for $m \geq 4$,

$$
\widetilde{\mathcal{U}} \in C^{\infty}\left(\mathcal{O}_{\delta} \times \widetilde{\mathcal{O}}_{\delta}^{m+\alpha}, h^{4+\alpha}\left(\mathbb{E}_{s}\right)\right) .
$$

Next, we further show some much more profound properties of $\widetilde{\mathcal{U}}$. Recall from Part II.1 of Hamilton [15, Banach space $h^{4+\alpha}(\mathbb{S})$ can be regarded as a tame Fréchet space, $C^{\infty}(\mathbb{S})$ with a collection of seminorms $\left\{\|\|_{h^{m+\alpha}(\mathbb{S})}, m=0,1,2 \cdots\right\}$, and $B U C^{\infty}(\mathbb{K})$ with a collection of seminorms $\left\{\|\|_{h^{m+\alpha}(\mathbb{K})}, m=0,1,2 \cdots\right\}$ are both tame Fréchet spaces. Denote

$$
\widetilde{\mathcal{O}}_{\delta}^{\infty}=\left\{\eta \in C^{\infty}(\mathbb{S}):\|\eta\|_{h^{4+\alpha}(\mathbb{S})}<\delta\right\} .
$$

By Theorem 3.3.5 in Part II of [15], we have

$$
\widetilde{\mathcal{U}} \text { is a smooth tame mapping from } \mathcal{O}_{\delta} \times \widetilde{\mathcal{O}}_{\delta}^{\infty} \text { to } h^{4+\alpha}\left(\mathbb{E}_{s}\right) \cap B U C^{\infty}(\mathbb{K}),
$$


which means that for all $m \in \mathbb{N}, m \geq 4$,

$$
\widetilde{\mathcal{U}} \in C^{\infty}\left(\mathcal{O}_{\delta} \times \widetilde{\mathcal{O}}_{\delta}^{m+\alpha}, h^{4+\alpha}\left(\mathbb{E}_{s}\right) \cap h^{m+\alpha}(\mathbb{K})\right),
$$

and

$$
\|\tilde{\mathcal{U}}(\rho, \eta)\|_{h^{4+\alpha}\left(\mathbb{E}_{s}\right)}+\|\tilde{\mathcal{U}}(\rho, \eta)\|_{h^{m+\alpha}(\mathbb{K})} \leq C_{m}\left(1+\|\rho\|_{h^{4+\alpha}(\mathbb{S})}+\|\eta\|_{h^{m+\alpha}(\mathbb{S})}\right),
$$

where $C_{m}$ is a positive constant dependent on $m$.

We define a mapping $F: \mathcal{O}_{\delta} \times \widetilde{\mathcal{O}}_{\delta}^{m+\alpha} \rightarrow h^{m+\alpha}(\mathbb{S})$ by

$$
F(\rho, \eta)=\left.\widetilde{\mathcal{U}}(\rho, \eta)\right|_{J_{s}}-\hat{\sigma}
$$

It is easy to see that $F \in C^{\infty}\left(\mathcal{O}_{\delta} \times \widetilde{\mathcal{O}}_{\delta}^{m+\alpha}, h^{m+\alpha}(\mathbb{S})\right)$. Moreover, by (3.12) we have

$$
F \text { is a smooth tame mapping from } \mathcal{O}_{\delta} \times \widetilde{\mathcal{O}}_{\delta}^{\infty} \text { to } C^{\infty}(\mathbb{S}) \text {. }
$$

Clearly, $F(0,0)=0$ and problem (3.9) is equivalent to the equation $F(\rho, \eta)=0$.

Next we compute the Fréchet derivative of $F$ with respect to $\eta$ at $(\rho, \eta) \in \mathcal{O}_{\delta} \times \widetilde{\mathcal{O}}_{\delta}^{\infty}$, which is denoted by $D_{\eta} F(\rho, \eta)$. Let $\mathcal{U}(\rho, \eta)$ be the solution of the first three equations of problem (3.9). For any $\zeta \in C^{\infty}(\mathbb{S})$, we easily verify that

$$
D_{\eta} F(\rho, \eta) \zeta=\left.\mathcal{Z}(\rho, \eta, \zeta)\right|_{J_{\eta}}
$$

where $z=\mathcal{Z}(\rho, \eta, \zeta)$ is the solution of the following problem

$$
\mathcal{A}(\rho) z=z \quad \text { in } \mathbb{E}_{\eta}, \quad z=0 \quad \text { on } \Gamma_{s}, \quad \partial_{y} z=-\partial_{y y} \mathcal{U}(\rho, \eta) \zeta \quad \text { on } J_{\eta} .
$$

Since $\mathcal{U}(0,0)=\left.\sigma_{s}\right|_{\mathbb{E}_{s}}$, we have $\left.\partial_{y y} \mathcal{U}(0,0)\right|_{J_{s}}=\sigma_{s}^{\prime \prime}\left(\eta_{s}^{+}\right)=\hat{\sigma}>0$. Thus for sufficiently small $\delta>0$, we have $\left.\partial_{y y} \mathcal{U}(\rho, \eta)\right|_{J_{\eta}}>\hat{\sigma} / 2$ for $(\rho, \eta) \in \mathcal{O}_{\delta} \times \widetilde{\mathcal{O}}_{\delta}^{\infty}$. By (3.16), for any $\xi \in C^{\infty}(\mathbb{S})$ we have

$$
\left[D_{\eta} F(\rho, \eta)\right]^{-1} \xi=-\left.\frac{\partial_{y} \mathcal{T}(\rho, \eta, \xi)}{\partial_{y y} \mathcal{U}(\rho, \eta)}\right|_{J_{\eta}}
$$

where $z=\mathcal{T}(\rho, \eta, \xi)$ is the solution of the problem

$$
\mathcal{A}(\rho) z=z \quad \text { in } \mathbb{E}_{\eta}, \quad z=0 \text { on } \Gamma_{s}, \quad z=\xi \quad \text { on } J_{\eta} .
$$

Notice that $D_{\eta} F(0,0)$ is an isomorphism from $h^{m+\alpha}(\mathbb{S})$ onto $h^{m+1+\alpha}(\mathbb{S})$ for all $m \in \mathbb{N}$, so classical implicit function theorem in Banach spaces is not available here. But on the other hand, similarly as (3.15), we can show the mapping

$$
(\rho, \eta, \xi) \mapsto\left[D_{\eta} F(\rho, \eta)\right]^{-1} \xi \text { is smooth tame from } \mathcal{O}_{\delta} \times \widetilde{\mathcal{O}}_{\delta}^{\infty} \times C^{\infty}(\mathbb{S}) \text { to } C^{\infty}(\mathbb{S}) .
$$

Thus by Nash-Moser implicit function theorem (see Theorem 3.3.1 in Part III of [15]), there exist sufficiently small $\delta_{1}, \delta_{1}^{\prime} \in\left(0, r_{0}\right)$, and a unique smooth tame mapping $\mathcal{S}$ from $\mathcal{O}_{\delta_{1}}$ to $\widetilde{\mathcal{O}}_{\delta_{1}^{\prime}}^{\infty}$ such that

$$
\mathcal{S}(0)=0 \quad \text { and } \quad F(\rho, \mathcal{S}(\rho))=0 .
$$


By letting $u=\mathcal{U}(\rho, \mathcal{S}(\rho))$ and $\eta=\mathcal{S}(\rho)$, we see that $(u, \eta)$ is the solution of problem (3.9), and the mapping $\rho \mapsto(u, \eta)$ is smooth. The proof is complete.

By the proof of Lemma 3.2, for any $\rho \in \mathcal{O}_{\delta_{1}}$, problem (3.8) has a unique solution

$$
u=\left\{\begin{array}{ll}
\mathcal{U}(\rho, \mathcal{S}(\rho)) & \text { in } \mathbb{E}_{\mathcal{S}(\rho)}, \\
\hat{\sigma} & \text { in } \mathbb{D}_{\mathcal{S}(\rho)},
\end{array} \quad \text { and } \quad \eta=\mathcal{S}(\rho) .\right.
$$

Next we consider the following problem

$$
\left\{\begin{aligned}
\mathcal{A}(\rho) v & =-\mu(u-\tilde{\sigma}) \chi_{\mathbb{E}_{\eta}}+\nu \chi_{\mathbb{D}_{\eta}} & & \text { in } \Omega_{s}, \\
v & =\gamma \mathcal{K}(\rho) & & \text { on } \Gamma_{s}, \\
\llbracket v \rrbracket & =0, \quad \llbracket \partial_{n} v \rrbracket=0 & & \text { on } J_{\eta}, \\
\partial_{y} v & =0 & & \text { on } \Gamma_{0},
\end{aligned}\right.
$$

where $u$ and $\eta$ are given by (3.17). For the sake of simplicity, we first study

$$
\left\{\begin{aligned}
\mathcal{A}(\rho) w^{+} & =-\mu(\mathcal{U}(\rho, \mathcal{S}(\rho))-\tilde{\sigma}) & & \text { in } \mathbb{E}_{\mathcal{S}(\rho)}, \\
\mathcal{A}(\rho) w^{-} & =\nu & & \text { in } \mathbb{D}_{\mathcal{S}(\rho)}, \\
w^{+} & =0 & & \text { on } \Gamma_{s} \\
w^{+} & =w^{-} & & \text {on } J_{\mathcal{S}(\rho)} \\
\partial_{n} w^{+} & =\partial_{n} w^{-} & & \text {on } J_{\mathcal{S}(\rho)} \\
\partial_{y} w^{-} & =0 & & \text { on } \Gamma_{0}
\end{aligned}\right.
$$

Lemma 3.4 There exists a positive constant $\delta_{2} \in\left(0, \delta_{1}\right)$ such that for any $\rho \in \mathcal{O}_{\delta_{2}}$, problem (3.19) has a unique solution $\left(w^{+}, w^{-}\right) \in h^{4+\alpha}\left(\mathbb{E}_{\mathcal{S}(\rho)}\right) \times h^{4+\alpha}\left(\mathbb{D}_{\mathcal{S}(\rho)}\right)$, and the mapping $\rho \mapsto\left(w^{+}, w^{-}\right)$is smooth in $\mathcal{O}_{\delta_{2}}$.

Proof. For given $\rho \in \mathcal{O}_{\delta_{1}}$ and $\zeta \in h^{4+\alpha}(\mathbb{S})$, we consider

$$
\left\{\begin{array} { r l } 
{ \mathcal { A } ( \rho ) w ^ { + } = - \mu ( \mathcal { U } ( \rho , \mathcal { S } ( \rho ) ) - \tilde { \sigma } ) } & { \text { in } \mathbb { E } _ { \mathcal { S } ( \rho ) , } , } \\
{ w ^ { + } = \zeta } & { \text { on } J _ { \mathcal { S } ( \rho ) } , } \\
{ w ^ { + } = 0 } & { \text { on } \Gamma _ { s } , }
\end{array} \quad \left\{\begin{array}{rl}
\mathcal{A}(\rho) w^{-}=\nu & \text { in } \mathbb{D}_{\mathcal{S}(\rho)}, \\
w^{-}=\zeta & \text { on } J_{\mathcal{S}(\rho)}, \\
\partial_{y} w^{-}=0 & \text { on } \Gamma_{0} .
\end{array}\right.\right.
$$

From Lemma 3.2, we see $\mathcal{S}(\rho) \in C^{\infty}(\mathbb{S})$ and $\mathcal{U}(\rho, \mathcal{S}(\rho)) \in h^{4+\alpha}\left(\mathbb{E}_{\mathcal{S}(\rho)}\right)$. By classical regularity theory of elliptic differential equations, problem (3.20) has a unique solution $\left(w^{+}, w^{-}\right)$such that

$$
w^{+}:=\mathcal{W}^{+}(\rho, \zeta) \in h^{4+\alpha}\left(\mathbb{E}_{\mathcal{S}(\rho)}\right) \quad \text { and } \quad w^{-}:=\mathcal{W}^{-}(\rho, \zeta) \in h^{4+\alpha}\left(\mathbb{D}_{\mathcal{S}(\rho)}\right)
$$

Since the mappings $\mathcal{S}$ and $\mathcal{U}$ are both smooth in $\mathcal{O}_{\delta_{1}}$, the mappings $\mathcal{W}^{+}$and $\mathcal{W}^{-}$are also smooth in $\mathcal{O}_{\delta_{1}} \times h^{4+\alpha}(\mathbb{S})$. 
Recall that $\partial_{n}$ is outward normal derivative on $J_{\mathcal{S}(\rho)}$ with respect to $\mathbb{E}_{\mathcal{S}(\rho)}$. Define a mapping $G: \mathcal{O}_{\delta_{1}} \times h^{4+\alpha}(\mathbb{S}) \rightarrow h^{3+\alpha}(\mathbb{S})$ by

$$
G(\rho, \zeta)=\left.\partial_{n} \mathcal{W}^{+}(\rho, \zeta)\right|_{J_{\mathcal{S}(\rho)}}-\left.\partial_{n} \mathcal{W}^{-}(\rho, \zeta)\right|_{J_{\mathcal{S}(\rho)}} \quad \text { for } \rho \in \mathcal{O}_{\delta_{1}}, \quad \zeta \in h^{4+\alpha}(\mathbb{S})
$$

It is easy to see that problem (3.19) is equivalent to the equation $G(\rho, \zeta)=0$.

Since $\mathcal{W}^{+}$and $\mathcal{W}^{-}$are smooth, we have

$$
G \in C^{\infty}\left(\mathcal{O}_{\delta_{1}} \times h^{4+\alpha}(\mathbb{S}), h^{3+\alpha}(\mathbb{S})\right)
$$

By (2.1) - (2.3), we see $G\left(0, p_{0}\right)=0$, where $p_{0}=p_{s}\left(\eta_{s}\right)$. Note that

$$
\mathcal{S}(0)=0, \quad \mathcal{U}(0, \mathcal{S}(0))=\sigma_{s}, \quad \mathcal{W}^{+}\left(0, p_{0}\right)=\left.p_{s}\right|_{\mathbb{E}_{s}}, \quad \mathcal{W}^{-}\left(0, p_{0}\right)=\left.p_{s}\right|_{\mathbb{D}_{s}} .
$$

By a direct computation, we have

$$
D_{\zeta} G\left(0, p_{0}\right) \xi=-\left.\partial_{y} z^{+}\right|_{J_{s}}+\left.\partial_{y} z^{-}\right|_{J_{s}} \quad \text { for } \quad \xi \in h^{4+\alpha}(\mathbb{S})
$$

where $z^{+}$and $z^{-}$are the solutions to the following two problems, respectively,

$$
\begin{aligned}
& \Delta z^{+}=0 \quad \text { in } \mathbb{E}_{s}, \quad z^{+}=\xi \quad \text { on } J_{s}, \quad z^{+}=0 \quad \text { on } \Gamma_{s}, \\
& \Delta z^{-}=0 \quad \text { in } \mathbb{D}_{s}, \quad z^{-}=\xi \quad \text { on } J_{s}, \quad \partial_{y} z^{-}=0 \quad \text { on } \Gamma_{0} \text {. }
\end{aligned}
$$

For any $\xi \in C^{\infty}(\mathbb{S})$ with the expression $\xi(x)=\sum_{k \in \mathbb{Z}} \xi_{k} e^{i k x}$, we obtain

$$
D_{\zeta} G\left(0, p_{0}\right) \xi=\sum_{k \in \mathbb{Z}} \tau_{k} \xi_{k} e^{i k x}
$$

where $\tau_{0}=\left(\rho_{s}-\eta_{s}\right)^{-1}$ and $\tau_{k}=k\left(\operatorname{coth} k\left(\rho_{s}-\eta_{s}\right)+\tanh k \eta_{s}\right)$ for $k \neq 0, k \in \mathbb{Z}$.

Obviously, there exist two positive constant $C_{1}$ and $C_{2}$ such that

$$
C_{1} \sqrt{k^{2}+1} \leq \tau_{k} \leq C_{2} \sqrt{k^{2}+1}
$$

It implies that

$$
D_{\zeta} G\left(0, p_{0}\right) \text { is an isomorphism from } H^{r+1}(\mathbb{S}) \text { onto } H^{r}(\mathbb{S}) \text { for } r>0 \text {, }
$$

where $H^{r}(\mathbb{S})=\left\{f \in L^{2}(\mathbb{S}): \sum_{k \in \mathbb{Z}}\left(k^{2}+1\right)^{r}|\widehat{f}(k)|^{2}<+\infty\right\}$.

From (3.25), we easily obtain that for $\xi \in C^{\infty}(\mathbb{S})$ with $\xi(x)=\sum_{k \in \mathbb{Z}} \xi_{k} e^{i k x}$,

$$
\left[D_{\zeta} G\left(0, p_{0}\right)\right]^{-1} \xi=\sum_{k \in \mathbb{Z}} \tau_{k}^{-1} \xi_{k} e^{i k x}
$$


Define a function $\tau(x)=x\left(\operatorname{coth}\left(\rho_{s}-\eta_{s}\right) x+\tanh \eta_{s} x\right)$ for $|x| \geq 1$. It is easy to verify that

$$
\tau_{k}=\tau(k) \quad \text { for } \quad k \neq 0 \quad \text { and } \quad \sup _{|x| \geq 1}\left|\tau^{\prime}(x)\right|+|x|\left|\tau^{\prime \prime}(x)\right|<+\infty .
$$

Using above relations one can prove that

$$
\left\{\begin{array}{l}
\sup _{k \in \mathbb{Z}}|k|\left|\frac{1}{\tau_{k}}\right|<+\infty, \\
\sup _{k \in \mathbb{Z}}|k|^{2}\left|\frac{1}{\tau_{k+1}}-\frac{1}{\tau_{k}}\right|<+\infty, \\
\sup _{k \in \mathbb{Z}}|k|^{3}\left|\frac{1}{\tau_{k+2}}-\frac{2}{\tau_{k+1}}+\frac{1}{\tau_{k}}\right|<+\infty .
\end{array}\right.
$$

Then by Theorem 4.5 of [2] (or [19]), we have

$$
\left[D_{\zeta} G\left(0, p_{0}\right)\right]^{-1} \in L\left(C^{r}(\mathbb{S}), C^{r+1}(\mathbb{S})\right) \quad \text { for } \quad r>0 .
$$

By Sobolev embedding theorem, $H^{4+r}(\mathbb{S}) \hookrightarrow C^{4+\alpha}(\mathbb{S})$ for $r>3 / 2$. Notice that $h^{4+\alpha}(\mathbb{S})$ is the closure of $H^{4+r}(\mathbb{S})$ in $C^{4+\alpha}(\mathbb{S})$ for $r>3 / 2$. By (3.26) and (3.27), we obtain that $\left[D_{\zeta} G\left(0, p_{0}\right)\right]^{-1} \in$ $L\left(h^{3+\alpha}(\mathbb{S}), h^{4+\alpha}(\mathbb{S})\right)$ and

$$
D_{\zeta} G\left(0, p_{0}\right) \text { is an isomorphism from } h^{4+\alpha}(\mathbb{S}) \text { onto } h^{3+\alpha}(\mathbb{S}) .
$$

Hence by classical implicit function theorem in Banach spaces, there exist sufficiently small constants $\delta_{2}, \delta_{2}^{\prime} \in\left(0, \delta_{1}\right)$, and a unique mapping $\mathcal{R} \in C^{\infty}\left(\mathcal{O}_{\delta_{2}}, h^{4+\alpha}(\mathbb{S})\right)$ such that

$$
\mathcal{R}(0)=p_{0}, \quad\left\|\mathcal{R}(\rho)-p_{0}\right\|_{h^{4+\alpha}(\mathbb{S})} \leq \delta_{2}^{\prime} \quad \text { and } \quad G(\rho, \mathcal{R}(\rho))=0 .
$$

By letting $\left(w^{+}, w^{-}\right)=\left(\mathcal{W}^{+}(\rho, \mathcal{R}(\rho)), \mathcal{W}^{-}(\rho, \mathcal{R}(\rho))\right)$, we see that $\left(w^{+}, w^{-}\right)$is the solution of problem (3.19), and the desired result follows immediately.

By the proof of Lemma 3.4, we denote

$$
\mathcal{W}(\rho)=\left\{\begin{array}{ll}
\mathcal{W}^{+}(\rho, \mathcal{R}(\rho)) & \text { in } \mathbb{E}_{\mathcal{S}(\rho)}, \\
\mathcal{W}^{-}(\rho, \mathcal{R}(\rho)) & \text { in } \mathbb{D}_{\mathcal{S}(\rho)},
\end{array} \quad \text { for } \quad \rho \in \mathcal{O}_{\delta_{2}}\right.
$$

Consider the problem

$$
\mathcal{A}(\rho) v_{0}=0 \quad \text { in } \Omega_{s}, \quad v_{0}=\gamma \mathcal{K}(\rho) \quad \text { on } \Gamma_{s}, \quad \partial_{y} v_{0}=0 \quad \text { on } \Gamma_{0} .
$$

Note that by (3.3), we have

$$
\mathcal{K} \in C^{\infty}\left(\mathcal{O}_{\delta_{2}}, h^{2+\alpha}(\mathbb{S})\right) .
$$

By classical regularity theory of elliptic differential equations, problem (3.29) has a unique solution $v_{0}:=\mathcal{V}(\rho) \in h^{2+\alpha}\left(\Omega_{s}\right)$. Moreover, by (3.5), (3.30) and Lemma 2.3 in 9],

$$
\mathcal{V} \in C^{\infty}\left(\mathcal{O}_{\delta_{2}}, h^{2+\alpha}\left(\Omega_{s}\right)\right)
$$


From (3.28), (3.29) and Lemma 3.4, for any $\rho \in \mathcal{O}_{\delta_{2}}$, we see problem (3.18) has a unique solution

$$
v=\mathcal{V}(\rho)+\mathcal{W}(\rho) \text {. }
$$

Later on, we always fix $0<\delta \leq \delta_{2}$. Define a mapping $\Psi: \mathcal{O}_{\delta} \rightarrow h^{1+\alpha}(\mathbb{S})$ by

$$
\Psi(\rho):=\mathcal{B}(\rho) \mathcal{V}(\rho)+\mathcal{B}(\rho) \mathcal{W}(\rho) \quad \text { for } \rho \in \mathcal{O}_{\delta} .
$$

It follows from (3.5), (3.31) and Lemma 3.4 that

$$
\Psi \in C^{\infty}\left(\mathcal{O}_{\delta}, h^{1+\alpha}(\mathbb{S})\right) .
$$

With all above reductions, we see that problem (3.7) is equivalent to the following Cauchy problem

$$
\begin{cases}\partial_{t} \rho+\Psi(\rho)=0 & \text { on } \mathbb{S}, t>0 \\ \rho(0)=\rho_{0} & \text { on } \mathbb{S} .\end{cases}
$$

More precisely, we have

Lemma 3.5 The function $\rho$ is the solution of problem (3.35) if and only if $(u, v, \eta, \rho)$ is the solution of problem (3.7) with $(u, v, \eta)$ given by (3.17) and (3.32).

Next we study local well-posedness of problem (3.35). For any $\rho \in \mathcal{O}_{\delta}$, we define the Fréchet derivative of nonlinear operator $\Psi$ at $\rho$ by

$$
D \Psi(\rho) \zeta:=\lim _{\varepsilon \rightarrow 0} \frac{\Psi(\rho+\varepsilon \zeta)-\Psi(\rho)}{\varepsilon} \quad \text { for } \zeta \in h^{4+\alpha}(\mathbb{S}) .
$$

Let $E_{0}$ and $E_{1}$ be two Banach spaces, $E_{1}$ is densely and continuously embedded into $E_{0}$. Denote by $\mathcal{H}\left(E_{1}, E_{0}\right)$ the subspace of all linear operators $A \in L\left(E_{1}, E_{0}\right)$ such that $-A$ generates a strongly continuous analytic semigroup on $E_{0}$. We have the following result:

Lemma 3.6 $D \Psi(\rho) \in \mathcal{H}\left(h^{4+\alpha}(\mathbb{S}), h^{1+\alpha}(\mathbb{S})\right)$ for $\rho \in \mathcal{O}_{\delta}$.

Proof. Let $\Psi_{1}(\rho):=\mathcal{B}(\rho) \mathcal{V}(\rho)$ and $\Psi_{2}:=\mathcal{B}(\rho) \mathcal{W}(\rho)$, then

$$
\Psi(\rho)=\Psi_{1}(\rho)+\Psi_{2}(\rho) \quad \text { for } \rho \in \mathcal{O}_{\delta} .
$$

Notice that the following problem is the corresponding transformed periodic Hele-Shaw model with surface tension:

$$
\left\{\begin{aligned}
\mathcal{A}(\rho) v_{0} & =0 & & \text { in } \Omega_{s}, t>0, \\
\partial_{y} v_{0} & =0 & & \text { on } \Gamma_{0}, t>0, \\
v_{0} & =\gamma \mathcal{K}(\rho) & & \text { on } \Gamma_{s}, t>0, \\
\rho_{t} & =-\mathcal{B}(\rho) v_{0} & & \text { on } \mathbb{S}, t>0,
\end{aligned}\right.
$$

and similarly, it can be reduced to $\partial_{t} \rho+\Psi_{1}(\rho)=0$ for $t>0$. Thus by well-known results of Hele-Shaw models (cf. 9]), we have $D \Psi_{1}(\rho) \in \mathcal{H}\left(h^{4+\alpha}(\mathbb{S}), h^{1+\alpha}(\mathbb{S})\right)$, for any $\rho \in \mathcal{O}_{\delta}$. 
On the other hand, by Lemma 3.4, (3.5) and (3.28), we have $\Psi_{2} \in C^{\infty}\left(\mathcal{O}_{\delta}, h^{3+\alpha}(\mathbb{S})\right)$ and $D \Psi_{2}(\rho) \in L\left(h^{4+\alpha}(\mathbb{S}), h^{3+\alpha}(\mathbb{S})\right)$. Since $h^{3+\alpha}(\mathbb{S})$ is compactly embedded into $h^{1+\alpha}(\mathbb{S})$, by the well-known perturbation result (cf. Theorem I.1.5.1 in [1], or Proposition 2.4.3 in [18]), we get the desired result.

The above result implies that problem (3.35) is of parabolic type in $\mathcal{O}_{\delta}$. Thus by using analytic semigroup theory and applications to parabolic differential problems (see [1] and [18]), we get the local well-posedness.

Theorem 3.7 Given $\rho_{0} \in \mathcal{O}_{\delta}$. There exists a maximal $T>0$ such that problem (3.35) has a unique solution $\rho \in C\left([0, T), \mathcal{O}_{\delta}\right) \cap C^{1}\left([0, T), h^{1+\alpha}(\mathbb{S})\right)$.

From Theorem 3.7, and combining Lemma 3.1, Lemma 3.5, we see that free boundary problem (1.1) is locally wellposed, and given $\rho_{0} \in \mathcal{O}_{\delta}$, there exists a unique solution $(\sigma, p, \eta, \rho)$ of problem (1.1).

\section{Linearization and Eigenvalues}

In this section we study linearization of problem (3.35) at the stationary solution $\rho=0$, and compute all eigenvalues of $D \Psi(0)$.

First, we study the linearization of free boundary problem (1.1) at flat stationary solution $\left(\sigma_{s}, p_{s}, \eta_{s}, \rho_{s}\right)$. Let

$$
\sigma=\sigma_{s}+\epsilon \phi(x, y, t), \quad p=p_{s}+\epsilon \psi(x, y, t), \quad \eta=\eta_{s}+\epsilon \xi(x, t), \quad \rho=\rho_{s}+\epsilon \zeta(x, t),
$$

where $\phi, \psi, \xi$ and $\zeta$ are unknown functions. At each time $t>0$, by (3.6), the mean curvature of the curve $y=\rho_{s}+\epsilon \zeta$ can be expressed by

$$
\mathcal{K}(\epsilon \zeta)=-\epsilon \zeta_{x x}+O\left(\epsilon^{2}\right)
$$

Let $\mathbf{n}_{\epsilon \zeta}=\left(-\epsilon \zeta_{x}, 1\right)$ be the outward normal direction on $y=\rho_{s}+\epsilon \zeta$. We compute

$$
\begin{aligned}
\left.\left\langle\nabla p, \mathbf{n}_{\epsilon \zeta}\right\rangle\right|_{y=\rho_{s}+\epsilon \zeta} & =\left.\left(-\epsilon p_{x} \zeta_{x}+p_{y}\right)\right|_{y=\rho_{s}+\epsilon \zeta}=\left.\partial_{y}\left(p_{s}+\epsilon \psi\right)\right|_{y=\rho_{s}+\epsilon \zeta}+O\left(\epsilon^{2}\right) \\
& =\epsilon p_{s}^{\prime \prime}\left(\rho_{s}\right) \zeta+\left.\epsilon \partial_{y} \psi\right|_{y=\rho_{s}}+O\left(\epsilon^{2}\right) \\
& =-\epsilon\left[\mu(\bar{\sigma}-\tilde{\sigma}) \zeta-\left.\partial_{y} \psi\right|_{y=\rho_{s}}\right]+O\left(\epsilon^{2}\right) .
\end{aligned}
$$

By substituting (4.1) into problem (1.1), collecting all first order $\epsilon$-terms and with the aid of (4.2), (4.3) and the fact that

$$
\sigma_{s}^{\prime \prime}\left(\eta_{s}^{+}\right)=\hat{\sigma}, \quad \sigma_{s}^{\prime \prime}\left(\eta_{s}^{-}\right)=0, \quad \sigma_{s}^{\prime}\left(\rho_{s}\right)=\sqrt{\bar{\sigma}^{2}-\hat{\sigma}^{2}}
$$




$$
p_{s}^{\prime \prime}\left(\eta_{s}^{+}\right)=-\mu(\hat{\sigma}-\tilde{\sigma}), \quad p_{s}^{\prime \prime}\left(\eta_{s}^{-}\right)=\nu, \quad p_{s}^{\prime}\left(\rho_{s}\right)=0,
$$

we obtain the linearization of problem (1.1) at $\left(\sigma_{s}, p_{s}, \eta_{s}, \rho_{s}\right)$ is given by

$$
\begin{cases}\Delta \phi=\phi \chi_{\mathbb{E}_{s}} & \text { in } \Omega_{s}, t>0, \\ \Delta \psi=-\mu \phi \chi_{\mathbb{E}_{s}} & \text { in } \Omega_{s}, t>0, \\ \phi=-\sqrt{\bar{\sigma}^{2}-\hat{\sigma}^{2}} \zeta, \quad \psi=-\gamma \zeta_{x x} & \text { on } \Gamma_{s}, t>0, \\ \phi=0, \quad \llbracket \partial_{y} \phi \rrbracket=-\hat{\sigma} \xi & \text { on } J_{s}, t>0, \\ \llbracket \psi \rrbracket=0, \quad \llbracket \partial_{y} \psi \rrbracket=(\mu(\hat{\sigma}-\tilde{\sigma})+\nu) \xi & \text { on } J_{s}, t>0, \\ \partial_{y} \phi=0, \quad \partial_{y} \psi=0 & \text { on } \Gamma_{0}, t>0, \\ \partial_{t} \zeta=-\left.\partial_{y} \psi\right|_{y=\rho_{s}}+\mu(\bar{\sigma}-\tilde{\sigma}) \zeta & \text { on } \mathbb{S}, t>0 .\end{cases}
$$

For any given $\zeta \in h^{4+\alpha}(\mathbb{S})$, by solving problem $(4.4)_{1}-(4.4)_{6}$, we get a unique solution $(\phi, \psi, \xi)$.

Since problem (1.1) is equivalent to problem (3.35), their corresponding linearizations at flat stationary solution are also equivalent. It implies that

$$
D \Psi(0) \zeta=\left.\partial_{y} \psi\right|_{y=\rho_{s}}-\mu(\bar{\sigma}-\tilde{\sigma}) \zeta \quad \text { for } \zeta \in h^{4+\alpha}(\mathbb{S})
$$

Next, we given an explicit expression of $D \Psi(0)$ and study its eigenvalues. For any given

$$
\zeta(x)=\sum_{k \in \mathbb{Z}} c_{k} \mathbf{e}^{i k x} \in C^{\infty}(\mathbb{S}),
$$

set

$$
\phi(x, y)=\sum_{k \in \mathbb{Z}} a_{k}(y) \mathbf{e}^{i k x}, \quad \psi(x, y)=\sum_{k \in \mathbb{Z}} b_{k}(y) \mathbf{e}^{i k x}, \quad \xi(x)=\sum_{k \in \mathbb{Z}} d_{k} \mathbf{e}^{i k x},
$$

where $a_{k}(y)$ and $b_{k}(y)$ are unknown functions, $d_{k}$ is unknown coefficient for each $k \in \mathbb{Z}$.

Substituting (4.6) and (4.7) into (4.4), we see that for each $k \in \mathbb{Z}$, there hold

$$
\begin{cases}a_{k}^{\prime \prime}-k^{2} a_{k}=a_{k} & \text { for } \eta_{s}<y<\rho_{s}, \\ a_{k}(y)=0 & \text { for } 0<y \leq \eta_{s}, \\ a_{k}^{\prime}\left(\eta_{s}^{+}\right)=-\hat{\sigma} d_{k}, & \\ a_{k}\left(\rho_{s}\right)=-\sqrt{\bar{\sigma}^{2}-\hat{\sigma}^{2}} c_{k}, & \end{cases}
$$


and

$$
\begin{cases}b_{k}^{\prime \prime}-k^{2} b_{k}=-\mu a_{k} & \text { for } \eta_{s}<y<\rho_{s} \\ b_{k}^{\prime \prime}-k^{2} b_{k}=0 & \text { for } 0<y<\eta_{s} \\ b_{k}^{\prime}\left(\eta_{s}^{+}\right)=b_{k}^{\prime}\left(\eta_{s}^{-}\right)+(\mu(\hat{\sigma}-\tilde{\sigma})+\nu) d_{k} & \\ b_{k}\left(\eta_{s}^{+}\right)=b_{k}\left(\eta_{s}^{-}\right), & \\ b_{k}\left(\rho_{s}\right)=\gamma k^{2} c_{k} & \\ b_{k}^{\prime}(0)=0 & \end{cases}
$$

By solving problem (4.8), we obtain that for each $k \in \mathbb{Z}$,

$$
a_{k}(y)= \begin{cases}-\frac{\sinh \sqrt{k^{2}+1}\left(y-\eta_{s}\right)}{\sinh \sqrt{k^{2}+1}\left(\rho_{s}-\eta_{s}\right)} \sqrt{\bar{\sigma}^{2}-\hat{\sigma}^{2}} c_{k} & \text { for } \eta_{s} \leq y \leq \rho_{s}, \\ 0 & \text { for } 0<y<\eta_{s},\end{cases}
$$

and

$$
d_{k}=\frac{\sqrt{k^{2}+1} \sqrt{\bar{\sigma}^{2}-\hat{\sigma}^{2}} c_{k}}{\hat{\sigma} \sinh \sqrt{k^{2}+1}\left(\rho_{s}-\eta_{s}\right)} .
$$

Then by solving problem (4.9), an elementary computation shows that, for each $k \neq 0, k \in \mathbb{Z}$,

$$
b_{k}(y)= \begin{cases}-\mu a_{k}(y)+\left(\gamma k^{2}-\mu \sqrt{\bar{\sigma}^{2}-\hat{\sigma}^{2}}\right) c_{k} \frac{\cosh k y}{\cosh k \rho_{s}}+e_{k} \frac{\sinh k\left(\rho_{s}-y\right)}{\sinh k\left(\rho_{s}-\eta_{s}\right)} & \text { for } \eta_{s} \leq y \leq \rho_{s}, \\ \left(\gamma k^{2}-\mu \sqrt{\bar{\sigma}^{2}-\hat{\sigma}^{2}}\right) c_{k} \frac{\cosh k y}{\cosh k \rho_{s}}+e_{k} \frac{\cosh k y}{\cos k \eta_{s}} & \text { for } 0<y<\eta_{s},\end{cases}
$$

where

$$
e_{k}=\frac{(\mu \tilde{\sigma}-\nu) d_{k}}{k\left[\operatorname{coth} k\left(\rho_{s}-\eta_{s}\right)+\tanh k \eta_{s}\right]} \quad \text { for } k \neq 0, k \in \mathbb{Z}
$$

By using (2.4) and (4.11), we have $d_{0}=c_{0}$, then

$$
b_{0}(y)= \begin{cases}{\left[\mu \sqrt{\bar{\sigma}^{2}-\hat{\sigma}^{2}}\left(\frac{\sinh \left(y-\eta_{s}\right)}{\sinh \left(\rho_{s}-\eta_{s}\right)}-1\right)+(\mu \tilde{\sigma}-\nu)\left(\rho_{s}-y\right)\right] c_{0}} & \text { for } \eta_{s} \leq y \leq \rho_{s}, \\ {\left[-\mu \sqrt{\bar{\sigma}^{2}-\hat{\sigma}^{2}}+(\mu \tilde{\sigma}-\nu)\left(\rho_{s}-\eta_{s}\right)\right] c_{0}} & \text { for } 0<y<\eta_{s} .\end{cases}
$$

By (4.10)-(4.13), for $k \neq 0$, we compute

$$
\begin{aligned}
& b_{k}^{\prime}\left(\rho_{s}\right)-\mu(\bar{\sigma}-\tilde{\sigma}) c_{k} \\
= & -\mu a_{k}^{\prime}\left(\rho_{s}\right)+\left(\gamma k^{2}-\mu \sqrt{\bar{\sigma}^{2}-\hat{\sigma}^{2}}\right) c_{k} k \tanh k \rho_{s}-\frac{k e_{k}}{\sinh k\left(\rho_{s}-\eta_{s}\right)}-\mu(\bar{\sigma}-\tilde{\sigma}) c_{k} \\
= & \lambda_{k}(\gamma) c_{k},
\end{aligned}
$$


where

$$
\begin{aligned}
\lambda_{k}(\gamma) & =\gamma k^{3} \tanh k \rho_{s}+\mu \sqrt{\bar{\sigma}^{2}-\hat{\sigma}^{2}}\left[\sqrt{k^{2}+1} \operatorname{coth} \sqrt{k^{2}+1}\left(\rho_{s}-\eta_{s}\right)-k \tanh k \rho_{s}\right] \\
& +\frac{(-\mu \tilde{\sigma}+\nu) \sqrt{k^{2}+1} \sqrt{\bar{\sigma}^{2}-\hat{\sigma}^{2}}}{\hat{\sigma} \sinh k\left(\rho_{s}-\eta_{s}\right) \sinh \sqrt{k^{2}+1}\left(\rho_{s}-\eta_{s}\right)\left[\operatorname{coth} k\left(\rho_{s}-\eta_{s}\right)+\tanh k \eta_{s}\right]}-\mu(\bar{\sigma}-\tilde{\sigma}),
\end{aligned}
$$

for $k \neq 0$ and $\gamma>0$.

Note that (2.4) implies $\operatorname{coth}\left(\rho_{s}-\eta_{s}\right)=\bar{\sigma} / \sqrt{\bar{\sigma}^{2}-\hat{\sigma}^{2}}$. Then from (4.14) we compute

$$
\begin{aligned}
b_{0}^{\prime}\left(\rho_{s}\right)-\mu(\bar{\sigma}-\tilde{\sigma}) c_{0} & =\mu c_{0} \sqrt{\bar{\sigma}^{2}-\hat{\sigma}^{2}} \operatorname{coth}\left(\rho_{s}-\eta_{s}\right)-(\mu \tilde{\sigma}-\nu) c_{0}-\mu(\bar{\sigma}-\tilde{\sigma}) c_{0} \\
& =\mu \bar{\sigma} c_{0}-(\mu \tilde{\sigma}-\nu) c_{0}-\mu(\bar{\sigma}-\tilde{\sigma}) c_{0} \\
& =\nu c_{0} .
\end{aligned}
$$

By (4.5)-(4.7) and (4.15)-(4.17), we have

Lemma 4.1 For any $\zeta \in C^{\infty}(\mathbb{S})$ given by $\zeta=\sum_{k \in \mathbb{Z}} c_{k} \mathbf{e}^{i k x}$, there holds

$$
D \Psi(0) \zeta=\sum_{k \in \mathbb{Z}} \lambda_{k}(\gamma) c_{k} \mathbf{e}^{i k x}
$$

where $\lambda_{k}(\gamma)$ is given by (4.16) for $k \neq 0$, and $\lambda_{0}(\gamma) \equiv \nu$.

Obviously, for each $k \in \mathbb{Z}$ and $\gamma>0, \lambda_{k}(\gamma)$ is an eigenvalue of the linearized operator $D \Psi(0)$. We have the following properties:

Lemma 4.2 (i) For any $\gamma>0$, $\lim _{k \rightarrow \infty} \lambda_{k}(\gamma)=+\infty$.

(ii) There exists a constant $\gamma_{*}>0$, such that if $\gamma>\gamma_{*}$, we have $\lambda_{k}(\gamma)>0$ for all $k \in \mathbb{Z}$; and if $0<\gamma<\gamma_{*}$, there exists at least an integer $k_{0} \in \mathbb{Z}$ such that $\lambda_{k_{0}}(\gamma)<0$.

Proof. (i) By a direct analysis, we have

$$
\begin{gathered}
\lim _{k \rightarrow+\infty} \tanh k \rho_{s}=\lim _{k \rightarrow+\infty} \operatorname{coth} k\left(\rho_{s}-\eta_{s}\right)=1, \\
\lim _{k \rightarrow-\infty} \tanh k \rho_{s}=\lim _{k \rightarrow-\infty} \operatorname{coth} k\left(\rho_{s}-\eta_{s}\right)=-1, \\
\lim _{k \rightarrow \infty}\left(\sqrt{k^{2}+1} \operatorname{coth} \sqrt{k^{2}+1}\left(\rho_{s}-\eta_{s}\right)-k \tanh k \rho_{s}\right)=0 .
\end{gathered}
$$

Hence by (4.16), we immediately obtain $\lim _{k \rightarrow \infty} \lambda(\gamma)=+\infty$ for any $\gamma>0$.

(ii) Define a sequence $\left\{\gamma_{k}\right\}_{k \neq 0}$ by

$$
\begin{aligned}
\gamma_{k} & :=\frac{1}{k^{3} \tanh k \rho_{s}}\left\{\mu \sqrt{\bar{\sigma}^{2}-\hat{\sigma}^{2}}\left[k \tanh k \rho_{s}-\sqrt{k^{2}+1} \operatorname{coth} \sqrt{k^{2}+1}\left(\rho_{s}-\eta_{s}\right)\right]\right. \\
& \left.+\frac{(\mu \tilde{\sigma}-\nu) \sqrt{k^{2}+1} \sqrt{\bar{\sigma}^{2}-\hat{\sigma}^{2}}}{\hat{\sigma} \sinh k\left(\rho_{s}-\eta_{s}\right) \sinh \sqrt{k^{2}+1}\left(\rho_{s}-\eta_{s}\right)\left[\operatorname{coth} k\left(\rho_{s}-\eta_{s}\right)+\tanh k \eta_{s}\right]}+\mu(\bar{\sigma}-\tilde{\sigma})\right\} .
\end{aligned}
$$


Clearly, we have

$$
\lim _{k \rightarrow \infty} \gamma_{k}=0 \quad \text { and } \quad \lim _{k \rightarrow \infty} k^{3} \tanh k \rho_{s} \gamma_{k}=\mu(\bar{\sigma}-\tilde{\sigma})>0
$$

Let

$$
\gamma_{*}:=\sup _{k \neq 0}\left\{\gamma_{k}\right\}
$$

By (4.20) we see that $\gamma_{*}$ is well-defined and $\gamma_{*}>0$.

By (4.19), we rewrite (4.16) as

$$
\lambda_{k}(\gamma)=k^{3} \tanh k \rho_{s}\left(\gamma-\gamma_{k}\right) \quad \text { for } k \neq 0, k \in \mathbb{Z}
$$

Then the desired result follows from (4.20) and (4.21).

Denote $\sigma(D \Psi(0))$ by the spectrum of $D \Psi(0)$. By Lemma 4.1 and Lemma 4.2, we have

Corollary 4.3 (i) If $\gamma>\gamma_{*}$, there exists a constant $\varpi>0$ such that

$$
\sigma(D \Psi(0)) \subset\{\lambda \in \mathbb{C}: \operatorname{Re} \lambda \geq \varpi\} .
$$

(ii) If $0<\gamma<\gamma_{*}$, then $\sigma(D \Psi(0)) \cap\{\lambda \in \mathbb{C}: \operatorname{Re} \lambda<0\} \neq \emptyset$.

Proof. Since $D \Psi(0) \in L\left(h^{4+\alpha}(\mathbb{S}), h^{1+\alpha}(\mathbb{S})\right)$, and $h^{4+\alpha}(\mathbb{S})$ is compactly embedded into $h^{1+\alpha}(\mathbb{S})$, we see that $\sigma(D \Psi(0))$ consists of all eigenvalues. By Lemma 4.1, we easily show that all eigenvalues of the restriction of $D \Psi(0)$ in $H^{4+r}(\mathbb{S})$ are given by $\lambda_{k}(\gamma)$ for $k \in \mathbb{Z}$. Since $h^{4+\alpha}(\mathbb{S})$ is the closure of $H^{4+r}(\mathbb{S})$ in $C^{4+\alpha}(\mathbb{S})$ for $r>3 / 2$, we have

$$
\sigma(D \Psi(0))=\left\{\lambda_{k}(\gamma) ; k \in \mathbb{Z}\right\}
$$

Let $\gamma>\gamma_{*}$. By (4.21) and (4.22), we see that

$$
\lambda_{k}(\gamma) \geq k^{3} \tanh k \rho_{s}\left(\gamma-\gamma_{*}\right) \geq \tanh \rho_{s}\left(\gamma-\gamma_{*}\right)>0 \quad \text { for } k \neq 0 .
$$

Notice that $\lambda_{0}(\gamma) \equiv \nu>0$. Take $\varpi \in\left(0, \min \left\{\tanh \rho_{s}\left(\gamma-\gamma_{*}\right), \nu\right\}\right)$, then $\lambda(\gamma)>\varpi$ for all $k \in \mathbb{Z}$. It implies that the assertion $(i)$ holds. The assertion $(i i)$ directly follows from Lemma $4.2(i i)$. The proof is complete.

\section{$5 \quad$ Asymptotic stability}

In this section we study asymptotic stability of the stationary solution $\rho=0$ of problem (3.35) and give a proof of our main result Theorem 1.2. 
Since problem (3.35) is of parabolic type in $h^{1+\alpha}(\mathbb{S})$, by using geometric theory of parabolic equations in Banach spaces, we have

Theorem 5.1 (i) If $\gamma>\gamma_{*}$, then the stationary solution 0 of problem (3.35) is asymptotically stable. More precisely, there exists a positive constant $\epsilon$ such that for any given $\rho_{0} \in \mathcal{O}_{\delta}$ with $\left\|\rho_{0}\right\|_{h^{4+\alpha}(\mathbb{S})}<\epsilon$, problem (3.35) has a unique solution $\rho(t) \in C\left([0,+\infty), \mathcal{O}_{\delta}\right) \cap C^{1}([0,+\infty)$, $\left.h^{1+\alpha}(\mathbb{S})\right)$, which converges exponentially fast to 0 as $t \rightarrow+\infty$.

(ii) If $0<\gamma<\gamma_{*}$, then the stationary solution 0 is unstable.

Proof. $\quad(i)$ Let $\gamma>\gamma_{*}$. Recall that $h^{4+\alpha}(\mathbb{S})$ is densely and compactly embedded into $h^{1+\alpha}(\mathbb{S})$. Set $A:=-D \Psi(0)$ and

$$
G(\rho):=-\Psi(\rho)+D \Psi(0) \rho \quad \text { for } \rho \in \mathcal{O}_{\delta} .
$$

Clearly, we have $G(0)=0$ and $D G(0)=0$. Problem (3.35) is equivalent to the following problem

$$
\rho^{\prime}(t)=A \rho(t)+G(\rho(t)) \quad \text { for } t>0, \quad \rho(0)=\rho_{0} .
$$

By Lemma 3.6, $A$ generates a strongly continuous analytic semigroup on $h^{1+\alpha}(\mathbb{S})$. By Corollary $4.3(i)$, we have $\sup \{\operatorname{Re} \lambda: \lambda \in \sigma(A)\}<-\varpi<0$. Thus by Theorem 9.1.2 of [18, there are positive constants $\omega, \epsilon$ and $M$ such that if the initial value $\rho_{0} \in \mathcal{O}_{\delta}$ and $\left\|\rho_{0}\right\|_{h^{4+\alpha}(\mathbb{S})}<\epsilon$, then the solution $\rho(t)$ of problem (3.35) exists globally and

$$
\|\rho(t)\|_{h^{4+\alpha}(\mathbb{S})}+\left\|\rho^{\prime}(t)\right\|_{h^{1+\alpha}(\mathbb{S})} \leq M e^{-\omega t}\left\|\rho_{0}\right\|_{h^{4+\alpha}(\mathbb{S})} \quad \text { for } t \geq 0 .
$$

(ii) If $0<\gamma<\gamma_{*}$, by Corollary $4.3($ ii $)$ we have $\sigma_{+}(A)=\sigma(A) \cap\{\lambda \in \mathbb{C}: \operatorname{Re} \lambda>0\} \neq \emptyset$ and $\inf \left\{\operatorname{Re} \lambda: \lambda \in \sigma_{+}(A)\right\}>0$. Thus by Theorem 9.1.3 in [18, the stationary solution $\rho=0$ is unstable. The proof is complete.

The proof of Theorem 1.2 By Lemma 3.1, Lemma 3.5 and Theorem $5.1(i)$, we see that the flat stationary solution $\left(\sigma_{s}, p_{s}, \eta_{s}, \rho_{s}\right)$ is asymptotically stable for $\gamma>\gamma_{*}$. More precisely, there is a constant $\epsilon>0$ such that for any $\rho_{0} \in \mathcal{O}_{\delta}$ satisfying $\left\|\rho_{0}\right\|_{h^{4+\alpha}(\mathbb{S})}<\epsilon$, problem (1.1) has a unique global solution $(\sigma(t), p(t), \eta(t), \rho(t))$ with the form of

$$
\sigma(t)=\Phi_{*}^{\tilde{\rho}(t)} u(t), \quad p(t)=\Phi_{*}^{\tilde{\rho}(t)} v(t), \quad \eta(t)=\eta_{s}+\mathcal{S}(\tilde{\rho}(t)), \quad \rho(t)=\rho_{s}+\tilde{\rho}(t),
$$

where $\tilde{\rho}(t)$ is the solution of problem (3.35) with $\tilde{\rho}(0)=\rho_{0}$, and $u(t), v(t), \mathcal{S}(\tilde{\rho}(t))$ are given by (3.17) and (3.32). By (5.2) and the reduction in Section 3, we see that $(\sigma(t), p(t), \eta(t), \rho(t))$ converges exponentially fast to $\left(\sigma_{s}, p_{s}, \eta_{s}, \rho_{s}\right)$ in $h^{4+\alpha}\left(\Omega_{\tilde{\rho}(t)} \backslash J_{\mathcal{S}(\tilde{\rho}(t))}\right) \times h^{2+\alpha}\left(\Omega_{\tilde{\rho}(t)} \backslash J_{\mathcal{S}(\tilde{\rho}(t))}\right) \times$ $h^{4+\alpha}(\mathbb{S}) \times h^{4+\alpha}(\mathbb{S})$, as time goes to infinity.

Similarly, by Lemma 3.1, Lemma 3.5 and Theorem 5.1 (ii), the flat stationary solution $\left(\sigma_{s}, p_{s}, \eta_{s}, \rho_{s}\right)$ is unstable for $0<\gamma<\gamma_{*}$. The proof is complete.

Remark 5.2 From (4.19) and (4.21), we easily obtain $d \gamma_{k} / d \nu<0$ for each $k \neq 0$, $k \in \mathbb{Z}$. Thus we have $d \gamma_{*} / d \nu \leq 0$. It implies that the smaller value of $\nu$ may make tumor more unaggressive. In the limiting case $\nu=0$, since $\lambda_{0}(\gamma)=\nu=0$, we have $0 \in \sigma(D \Psi(0))$. It implies that the flat stationary solution is not asymptotically stable any more for all $\gamma>0$. 


\section{References}

[1] H. Amann, Linear and Quasilinear Parabolic Problems, Birkhäuser, Basel (1995).

[2] W. Arendt and S. Bu, Operator-valued Fourier multipliers on periodic Besov spaces and applications, Proc. Edinb. Math. Soc., 47 (2004), 15-33.

[3] H. Byrne and M. Chaplain, Growth of necrotic tumors in the presence and absence of inhibitors, Math. Biosci., 135 (1996), 187-216.

[4] L. A. Caffarelli, The regularity of free boundaries in higher dimension, Acta Math., 139 (1977), $155-184$.

[5] S. Cui, Linearized stability theorem for invariant and quasi-invariant parabolic differential equations in Banach manifolds with applications to free boundary problems, (2016), arXiv:1606.09393.

[6] S. Cui and J. Escher, Bifurcation analysis of an elliptic free boundary problem modeling stationary growth of avascular tumors, SIAM J. Math. Anal., 39 (2007), 210-235.

[7] S. Cui and J. Escher, Asymptotic behavior of solutions of a multidimensional moving boundary problem modeling tumor growth, Comm. Partial Differential Equations, 33 (2008), 636-655.

[8] S. Cui and J. Escher, Well-posedness and stability of a multi-dimensional tumor growth model, Arch. Rational Mech. Anal., 191 (2009), 173-193.

[9] J. Escher and G. Simonett, Classical solutions for Hele-Shaw models with surface tension, Adv. Differential Equations, 2 (1997), 619-642.

[10] A. Friedman, Mathematical analysis and challenges arising from models of tumor growth, Math. Models Methods Appl. Sci., 17 (2007), suppl., 1751-1772.

[11] A. Friedman and B. Hu, Bifurcation from stability to instability for a free boundary problem arising in a tumor model, Arch. Rational Mech. Anal., 180 (2006), 293-330.

[12] A. Friedman and B. Hu, Asymptotic stability for a free boundary problem arising in a tumor model, J. Differential Equations, 227 (2006), 598-639.

[13] A. Friedman and B. Hu, Stability and instability of Liapunov-Schmidt and Hopf bifurcation for a free boundary problem arising in a tumor model, Trans. Amer. Math. Soc., 360 (2008), 5291-5342.

[14] A. Friedman and F. Reitich, Analysis of a mathematical model for the growth of tumors, J. Math. Biol., 38 (1999), 262-284.

[15] R. S. Hamilton, The inverse function theorem of Nash and Moser, Bull. Amer. Math. Soc., 7 (1982), $65-222$.

[16] W. Hao, J. D. Hauenstein, B. Hu and et al, Bifurcation for a free boundary problem modeling the growth of a tumor with a necrotic core, Nonliear Anal. Real World Appl., 13 (2012), 694-709.

[17] D. Kinderlehrer and G. Stampacchia, An introduction to variational inequalities and their applications, Pure and Applied Mathematics, 88. Academic Press, Inc, New York-London (1980).

[18] A. Lunardi, Analytic Semigroups and Optimal Regularity in Parabolic Problems, Birkhäuser, Basel (1995).

[19] H. Schmeisser and H. Triebel, Topics in Fourier Analysis and Function Spaces, John Wiley and Sons, New York (1987). 
[20] J. Wu, Stationary solutions of a free boundary problem modeling the growth of tumors with GibbsThomson relation, J. Differential Equations, 260 (2016), 5875-5893.

[21] J. Wu and S. Cui, Asymptotic behavior of solutions for parabolic differential equations with invariance and applications to a free boundary problem modeling tumor growth, Discrete Contin. Dyn. Syst., 26 (2010), 737-765.

[22] J. Wu and F. Zhou, Asymptotic behavior of solutions of a free boundary problem modeling tumor spheroid with Gibbs-Thomson relation, J. Differential Equations, 262 (2017), 4907-4930.

[23] F. Zhou, J. Escher and S. Cui, Bifurcation for a free boundary problem with surface tension modeling the growth of multi-layer tumors, J. Math. Anal. Appl., 337 (2008), 443-457. 